\section{Mobilizing variation}

piRNAs are small RNAs involved in transposon regulation in the germline. New work from Bozzetti and colleagues suggests that piRNAs may be involved in inducing phenotypic variation, which had been previously linked to Hsp90 in both Drosophila melanogaster and Arabidopsis thaliana. Whereas phenotypic stability would be favorable under stable

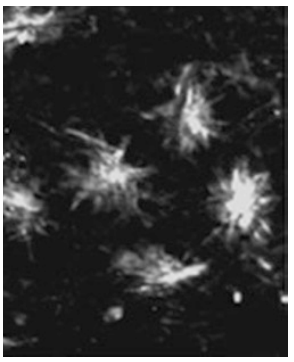
environmental conditions, phenotypic variation might be advantageous under conditions of stress, allowing beneficial and heritable traits to be revealed. Canalization is the idea that phenotypic variation is buffered under stable environmental conditions, though genetic variation might be present. Under conditions of stress, if such 'buffers' were diminished, varying traits dependent on underlying genetic variation would become apparent. In recent years, experimental evidence has emerged suggesting that Hsp90 might act as such a buffer, limiting phenotypic variation under stable conditions but allowing it to be revealed under stress. The authors have now found that under inhibition of hsp 83 in D. melanogaster, heritable phenotypic variation might be dependent on transposon mobilization through decreased activity of the piRNA pathway. When hsp 83 activity is decreased, either by using hsp 83 mutant strains or by applying the inhibitor geldanamycin, expression of Stellate repeat elements in testes cells is increased, as seen by transcript levels and protein crystalline aggregates (formed by a protein encoded within Stellate). As with other repetitive sequences, Stellate is repressed by a piRNA-mediated mechanism that also silences transposons. Indeed, transcription from a number of transposons increases under conditions of low hsp83 activity, whereas that of piRNAs decreases and phenotypic variation in progeny increases. The authors correlated these changes with differences in transposon positioning in parental strains compared to their progeny. In fact, when the authors examined a variant $\mathrm{F} 1$ strain that carries a phenotype resembling the Scutoid mutant, they found that this genomic locus is disrupted by an inserted $I$ transposable element. Although the mechanism by which piRNAs are affected by hsp 83 activity is currently unclear, this work links the piRNA pathway and increased transposition to phenotypic variation and argues that these factors together might provide the molecular basis for a classical evolutionary concept. (Nature doi:10.1038/nature08739, published online 10 January 2010)

SL

\section{Targeting DNA}

In the budding yeast Saccharomyces cerevisiae, some genes move from the nucleoplasm to the nuclear periphery upon transcriptional activation. One gene known to behave in this fashion is INO1, which encodes the enzyme inositol-1-phosphate synthase and is activated by inositol starvation and other stress conditions, but how this or any other gene is targeted to the nuclear periphery was unclear. Brickner and colleagues have now identified DNA elements within the INO1 promoter that are required for its nuclear periphery targeting, which they named gene-recruitment sequences (GRS). Using an array of lacO repeats and a LacI-GFP fusion, the authors tagged the INO1 gene in an ectopic locus to follow its subnuclear localization and then made deletions within the promoter region to identify the region(s) involved in targeting. This approach identified an 8-base-pair sequence (GRS I) that is sufficient to target the ectopic locus to the nuclear periphery; another sequence (GRS II) was identified further upstream in the endogenous INO1 locus that could also promote INO1 localization to the nuclear periphery. Mutation of both GRS I and

Written by Inês Chen, Angela K. Eggleston \& Sabbi Lall
GRS II blocked targeting of INO1 to the nuclear periphery and led to a defect in transcription. When the authors queried the yeast genome for GRS I, they found the sequence in many genes involved in heat shock and ER stress responses, conditions that also activate INO1. Among those genes is TSA2, which encodes a thioredoxin peroxidase; the authors found that TSA2 localizes to the nuclear periphery under oxidative stress and that this process is dependent on the GRS I in its promoter region. They also found that components of the nuclear pore complex are required for INO1 recruitment to the nuclear periphery, indicating that GRS elements might interact with the nuclear pore complex, an idea that is further supported by chromatin immunoprecipitation analysis. Finally, when the GRS was moved to the $3^{\prime}$ end of INO1, the gene localized to the nuclear periphery in a constitutive manner but transcription activation was defective, indicating that targeting of the gene promoter (rather than the gene itself) to the nuclear periphery seems to be important for transcriptional activation. Interestingly, GRS I seems to work in the distantly related fission yeast Schizosaccharomyces pombe, suggesting that this mechanism is evolutionarily ancient and well conserved. Exactly how the localization of the promoter to the nuclear periphery results in full transcriptional activation remains to be determined, but the identification of such DNA 'zip codes' furthers our insight into how gene-expression regulation can be encoded. (Nat. Cell. Biol. doi:10.1038/ncb2011, published online 24 January 2010)

IC

\section{The complexities of Aire}

In order to eliminate self-reactive $T$ cells, the immune system directs the production of peripheral-tissue self antigens (PTAs). The transcription factor Aire regulates expression of PTA genes. However, although Aire has some characteristics of a canonical transcription factor, there were indications that its mechanism of function is more complex than simple binding to a specific promoter sequence. To gain better insight into Aire's mechanism, Abramson et al. have identified Aire-associated proteins and clustered them by function. The most notable clusters were multiprotein complexes involved in chromatin structure or binding, RNAP II postinitiation events and pre-mRNA processing. Knockdown of members of these groups showed an effect on Aire-dependent transcription. One Aire-associated protein, the PI3 kinase DNA-PKcs, is mutated in a SCID mouse mutant, in which the authors found a reduction in both Aire-responsive PTA gene transcription and central tolerance response. The mechanism of action of another Aire interactor, the topoisomerase Top2a, involves the transient introduction of DNA double-strand breaks (DSBs). Surprisingly, knockdown experiments showed that Aire seems to interfere with Top2a's religation activity, resulting in elevated DSB levels in Aire-proficient cells. Aire also promotes high levels of mature, processed PTA transcripts, consistent with its interaction with pre-mRNA splicing factors; this effect is not a result of enhanced stabilization of the transcripts, so it is likely a direct effect on pre-mRNA processing. The authors propose that Aire targets regions of low transcriptional activity, using its ability to interact with histone $\mathrm{H} 3 \mathrm{~K} 4$ tails, and then promotes the introduction of DSBs by acting on Top2a. These DSBs would recruit repair factors and chromatin remodeling complexes, opening up the chromatin for transcription. Other Aire interactors may influence transcription more directly by facilitating removal of histones ahead of RNAPII and pre-mRNA splicing. Though this model is provocative, further validation is required to confirm the molecular details. (Cel/ 140,123-135, 2010) 